\title{
Efectividad de la vacuna antineumocócica en pacientes mayores de 65 años
}

A. Vila Córcoles, O. Ochoa Gondar, I. Hospital Guardiola, M. L. Marín Canseco, I. Guinea Oruechevarria, M. Álvarez Luy, Grupo de Estudio

EVAN-65

Centro de Atención Primaria Valls. Servicio Atención Primaria. Institut Catalá de la Salut. Tarragona-Valls. Tarragona

\section{RESUMEN}

Fundamentos: la efectividad de la vacuna antineumocócica de 23 serotipos (VAN 23S) respecto a la prevención de la adquisición de la infección está actualmente en discusión, y ésta además parece disminuir en la población anciana. Sin embargo, la indicación de la vacuna sigue manteniéndose en este grupo de edad. El objetivo de nuestro estudio fue evaluar la efectividad de la vacuna antineumocócica en dos cohortes (vacunados y no vacunados) de personas mayores de 65 años mediante comparación de tasas de incidencia y nivel de severidad de las neumonías en ambas cohortes.

Métodos: estudio de cohortes retrospectivo realizado en una Área Básica de Salud urbana (ABS), sobre dos cohortes de personas mayores de 65 años: 301 vacunados (CV) en 1993 y 301 no vacunados (CNV) hasta la actualidad. Se identificaron todas las neumonías acaecidas durante el periodo 1994-1999, calculando tasas de incidencia, grado de severidad e índice de letalidad.

Resultados: la tasa de incidencia media anual de neumonía fue de 13,29\%o en personas mayores de 65 años (16,6\% en CV y 9,96\%o en CNV), siendo el riesgo relativo para la cohorte vacunada de 1,67(IC 95\%: 0,95-2,92). No observamos diferencias significativas en cuanto al índice de letalidad
Effectiveness of pneumococcal vaccine in patients aged 65 years or older

\section{ABSTRACT}

Backgrounds: the effectiveness of the antipneumococcal vaccination (VAN $23 S$ ) regarding the prevention of a pneumococcal infection is currently controversial and appears to vanish among elderly people. However, vaccination continues to be indicated in this age group. The aim of this survey is to assess the effectiveness of this vaccine in two cohorts (vaccinated people and not vaccinated) among subjects elder than 65 by comparing incidence rates and severity degree of pneumonias in both groups.

Methods: retrospective cohort study realized in a Basic Health Care Area in two cohorts of patients older than 65 years: 301 were vaccinated and 301 non-vaccinated from 1993 to our days. All the pneumonias during the period 1994-1999, were identified and the incidence rate, severity degree and letal index were calculated.

Results: the mean incidence rate of pneumonia was 13,29\% in habitants in the 6-year period of time $(16,6 \%$ in VC and 9,96\% in NVC), with a relative risk for the vaccinated cohort of 1,67 (95\%CI: 0,95-2,92). No statistical significant differences were encountered neither in death rates in both groups (VC: 13.7 vs $15.7 \%$ in NVC) nor in the severity degree of pneumonias in both groups.

Trabajo preliminar del Estudio EVAN-65 (Efectividad de la vacunación antineumocócica sistemática entre la población mayor de 65 años) subvencionado con Beca del Fondo de Investigaciones Sanitarias (Exp, PI 021117). 
(CV 13,7\% frente a 15,7\% en CNV) y el grado de severidad en las neumonías de ambas cohortes.

Conclusiones: en nuestro ámbito no se ha podido demostrar un efecto protector de la VAN en $\mathrm{CV}$ respecto a $C N V$ ante la infección y tampoco se ha evidenciado efecto sobre la disminución de la severidad en las neumonías acaecidas en $C V$.

Palabras clave: Vacunación antineumocócica. Neumonía adquirida en la comunidad. Efectividad.
Conclusions: a protective effect of the antipneumococcal vaccination has not been proven in our country, neither related to the infection onset nor related to the severity acquisition of the pneumococcal infection.

Key words: Pneumococcal vaccines. Community-acquired pneumonia. Effectiveness.

\section{INTRODUCCIÓN}

La neumonía por Streptococcus pneumoniae representa del 20-30\% de las neumonías adquiridas en la comunidad en nuestro país, de las cuales $5-20 \%$ desarrollan bacteriemia ${ }^{1,2}$, siendo este agente responsable del $30-50 \%$ de las neumonías graves que requieren hospitalización, con una letalidad global del 5\% y del 15 al $20 \%$ en las formas bacteriémicas (ascendiendo al $60 \%$ en pacientes con enfermedades subyacentes y/o mayores de 70 años) ${ }^{3,4}$. En Cataluña se declaran entre 400 y 600 casos de neumonía por 100.000 habitantes/año, estimándose que la incidencia real en personas mayores de 65 años puede situarse entre 15 y $25 \%$ o $^{5,6}$.

La infección por Streptococcus pneumoniae debe abordarse desde dos frentes:

-Antibioterapia específica, que no ha logrado ser totalmente satisfactoria porque no se ha conseguido disminuir la mortalidad en los 3 primeros días de enfermedad, y además el porcentaje de neumococos resistentes a antibióticos de uso habitual ha aumentado $^{7-10}$.

- Inmunización activa con la vacuna inactivada; aunque inicialmente parecía claro su efecto reductor sobre el riesgo de infección sistémica y/o mortalidad, su efectividad respecto a la prevención de la adquisición de la infección está actualmente en discusión, y ésta además parece disminuir en grupos de alto riesgo y en la población anciana, especialmente después de los 75 años, ya que la respuesta de los anticuerpos es menor y sus niveles disminuyen más rápidamente ${ }^{10-12}$.

Estos datos están basados en estudios realizados hasta la fecha que difieren unos de otros sin llegar a conclusiones concretas. Parece que la efectividad de la vacuna en prevención de la enfermedad invasiva oscila entre $41-80 \%$, reduciéndose ésta en inmunodeprimidos y ancianos. Así, Forrester (1984, caso-control) observó nula protección pero con una muestra pequeña ${ }^{13}$. Butler (cohortes indirectas, 1993) concluyó que no existen diferencias en cuanto a efectividad en inmunocompetentes e inmunodeprimidos ${ }^{14}$, en contra Shapiro (caso-control, 1991) no demuestra protección en estos últimos pacientes ${ }^{15}$.

Un metaanálisis publicado en el año $2000^{16}$ revisaba trece estudios retrospectivos que incluían 45.000 sujetos; tres de ellos incluían 21.152 personas inmunocompetentes en los cuales la VAN fue efectiva para reducir la incidencia de neumonía de cualquier etiología (RR 0,47-0,66), de neumonía neumocócica (RR 0,11-0,23), letalidad por neumonía (RR 0,50-0,96) y bacteriemia (RR 0,09-0,34). Sin embargo, este mismo metaanálisis mostró que en diez estudios con 24.000 sujetos ancianos o con déficits inmunitarios, la vacuna no tuvo ningún efecto. Las últimas revisiones realizadas en nuestro país concluyen que no existe evidencia suficiente para recomendar la vacunación universal a todos los sujetos mayores de 65 años y que estaría justificada en ancianos institucionalizados o afectados de enfermedades pulmonares crónicas ${ }^{17,18}$.

Sin embargo, a pesar de las dudas existentes sobre la efectividad de la vacuna y la duración de su efecto, la pertinencia de la vacunación, la selección de la población diana y la conveniencia o no de la revacunación ${ }^{18}$, el Center for Disease Control de Atlanta $^{19,20}$, U.S. Preventive Service Task Force ${ }^{21}$ y los Departamentos de Sanidad y Salud Pública de nuestro país ${ }^{1}$, aconsejan la vacunación a un amplio grupo de población diana: mayores de 65 años en general y menores de 65 años con determinados factores de riesgo, existiendo una recomendación clara en las Comunidades de Galicia y Cataluña ${ }^{6,12}$.

El objetivo de nuestro estudio es analizar la incidencia de neumonías adquiridas en la comunidad y nivel de severidad de las mismas durante un periodo de seguimiento de 6 años en sendas cohortes (vacunados y no vacunados) compuestas por población general mayor de 65 años.

\section{MATERIAL Y MÉTODOS}

Diseño: estudio de cohortes retrospectivo en el ámbito de Atención Primaria de Salud (APS). Em- 
plazado en un área básica de 20.000 habitantes, que engloba una población de personas mayores de 65 años aproximada de 3.000 .

Población de estudio: estudiamos dos cohortes de 301 personas cada una (riesgo alfa $=0,05$; riesgo relativo esperado $=0,5$; riesgo beta estimado $=0,25)^{22}$.

- Cohorte vacunada (CV): cohorte de 301 personas mayores de 65 años (nacidos antes de 1928) vacunadas en 1993 con VAN 23S. Estos pacientes fueron seleccionados aleatoriamente del total de 930 mayores de 65 años vacunados en una campaña masiva ligada a la campaña antigripal desarrollada entre octubre y diciembre de 1993. Al tratarse de una evaluación retrospectiva, la selección de estos pacientes se efectuó en enero de 2000. Realizamos un muestreo aleatorio sistemático sobre el listado informatizado del total de pacientes que en enero de 1994 tenían más de 65 años $(\mathrm{n}=3016)$ y que constaban en el listado específico de pacientes vacunados mayores de 65 años asignados al ABS en aquella fecha (enero de 1994).

Una vez realizado el muestreo se procedió a la localización de las historias clínicas de Atención Primaria (HCAP) en los ficheros actuales de HCAP activas del centro. Aquellos pacientes seleccionados para la cohorte que ya no constaban como activos en el registro actual de HCAP del centro fueron localizados en archivo de pasivos (defunciones y traslados). Se incluyeron finalmente en la cohorte aquellos pacientes seleccionados inicialmente por el muestreo aleatorio aunque hubiesen fallecido (por cualquier causa) durante el periodo de estudio. En cambio los pacientes que hubieran causado baja por traslado de domicilio fueron sustituidos mediante un listado aleatorio de HCAP reservas.

- Cohorte no vacunada (CNV): cohorte de 301 personas mayores de 65 años no vacunadas hasta la actualidad. Esta cohorte está compuesta por el total de personas que, habiendo nacido antes de 1928, no fueron vacunadas durante la campaña masiva de 1993 ni en los años posteriores (ya sea por no haber acudido al centro durante los periodos de campaña, o bien por haberse negado a la vacunación). Del mismo modo que en la cohorte vacunada, en esta cohorte se hallan incluidos aquellos pacientes que no estando previamente vacunados, hubieran fallecido por cualquier causa durante el periodo de estudio. Quedaron excluidos aquellos pacientes que hubiesen causado baja por traslado de domicilio durante los años 1994-99.

Periodo de seguimiento: 6 años (enero 1994-diciembre 1999).

Fuente de datos: registro específico del programa de vacunación antineumocócica y revisión exhaustiva de las HCAP y de las historias clínicas del hospital de referencia (registro de neumonías ingresadas y registro de neumonías detectadas en el servicio de urgencias).
Variables: se definió "neumonía adquirida en la comunidad" como cuadro clínico-exploratorio compatible con proceso infeccioso respiratorio bajo, exigiéndose necesariamente la presencia de infiltrado radiológico de nueva presentación ${ }^{23}$.

Edad, sexo, presencia o no de factores de riesgo asociados a mayor susceptibilidad para adquirir infección neumocócica (diabetes mellitus, inmunodepresión (incluidos procesos neoplásicos activos), cardiopatía, EPOC, tabaquismo, enolismo, esplenectomía) 4 .

Medida del efecto: cálculo de la tasa de incidencia anual y global acumulada (1994-1999) de neumonías (sin poder precisar con exactitud la incidencia exacta de infección neumocócica en ambas cohortes que suponemos similar a la incidencia en nuestra zona) $)^{5,24}$.

Medida de la severidad: como primera medida de severidad valoramos el índice de letalidad en ambas cohortes tomando como fuente de datos las historias de Atención Primaria y del hospital de referencia. Asimismo valoramos de forma separada y conjunta una serie de 18 parámetros relacionados con la severidad (conformando una escala ordinal $0-18$, según presencia o no de criterio) agrupados en cuatro dimensiones: constantes clínicas en momento de diagnóstico, criterios de laboratorio, hallazgos radiológicos y tipo/duración del tratamiento (Tabla I).

Se trata de una escala de severidad de elaboración propia que posee validez de construcción-contenido, y que posee también validez de criterio si comparamos las puntuaciones obtenidas en la escala en función de la letalidad o no de los cuadros neumónicos.

Los anteriores criterios cuya existencia no constaba en HCAP ni en la historia hospitalaria (por haber sido diagnosticada y tratada exclusivamente desde APS) fueron valorados como ausentes.

El tratamiento estadístico se realizó mediante el programa SPSS-PC, facilitando las diferentes proporciones y medias observadas con sus correspondientes intervalos de confianza (IC) al 95\%. Asimismo se calculó el riesgo relativo (RR) con su correspondiente IC al 95\%. Para la asociación de variables cualitativas realizamos la prueba de Chi cuadrado y para la comparación de variables cuantitativas realizamos la prueba $t$ de Student, considerando diferencias significativas (ds) si $p<0,05$.

\section{RESULTADOS}

En la tabla II puede verse la distribución de edad, sexo y presencia de factores de riesgo en ambas cohortes. Respecto a la edad no existen diferencias significativas siendo la media 72,5 años en cohorte de vacunados y 72 años en no vacunados; 


\section{Tabla I}

\section{ESCALA DE SEVERIDAD DE LAS NEUMONÍAS}

\section{Escala de severidad}

Constantes clínicas

Alteración del nivel de consciencia

Temperatura $>38^{\circ} \mathrm{C}$

Frecuencia cardiaca $>120 \mathrm{Ipm}$

Frecuencia respiratoria $>30 \mathrm{rpm}$

Presión arterial sistólica $<90 \mathrm{mmHg}$

Criterios de laboratorio

Leucocitos $>15.000 / \mathrm{mm}^{3} 0<4.000 / \mathrm{mm}^{3} \quad+1$

Neutrófilos $>90 \%$

$\mathrm{PO}_{2}<60$ mmhg

Saturación de $\mathrm{O}_{2}<80 \%$

Hallazgos radiológicos

Afectación multilobar

Derrame pleural

Empiema

Cavitación

Extensión rápida (aumento del tamaño de los infiltrados $>50 \%$ respeto a Rx previas) +1

Tratamiento

Días de ingreso $>7$

Días de tratamiento endovenoso $>4$

Descompensación de patología previa

en cuanto al sexo, aunque existe ligero predominio femenino tampoco existen diferencias en la composición de ambas cohortes $(p=0,35)$.

En referencia a la presencia de factores de riesgo destaca únicamente el mayor porcentaje de fumadores en la cohorte de vacunados $(\mathrm{p}<0,05)$.

Entre las 602 personas estudiadas, observamos un total de 133 fallecimientos ( 68 en CV y 65 en CNV), lo que supuso una tasa media de mortalidad de 37,6 por mil personas/año durante el periodo de seguimiento en CV y de 35,9 por mil en CNV. Las pérdidas ocasionadas por la mortalidad general observada comportaron que el total de personas/año a riesgo de contraer neumonía fue de 1.602 en $\mathrm{CV}$, frente a un total de 1.611 personas/año a riesgo durante todo el periodo de seguimiento en CNV.

En la tabla III puede verse el número de perso-
Tabla II

\section{DESCRIPCIÓN DE LAS COHORTES DEL ESTUDIO}

\begin{tabular}{lcc}
\hline & Vacunados & No vacunados \\
\hline Edad (1993) & $72,5($ ds 7,7$)$ & $72,0($ DS 7,6$)$ \\
Sexo: mujeres & $57,5 \%(173)$ & $55,9 \%(168)$ \\
$\quad$ varones & $42,5 \%(128)$ & $44,1 \%(133)$ \\
Diabetes & $20,1 \%(60)$ & $17,5 \%(53)$ \\
Inmunodepresión & $1 \%(3)$ & $0,6 \%(2)$ \\
Cardiopatía & $22 \%(66)$ & $22,8 \%(68)$ \\
EPOC & $15 \%(45)$ & $14,2 \%(42)$ \\
Tabaquismo $\left(^{*}\right)$ & $10 \%(30)$ & $4,6 \%(14)$ \\
Enolismo & $6 \%(18)$ & $5,6 \%(17)$ \\
Esplenectomía & $0,3 \%(1)$ & $0,3 \%(1)$ \\
\hline (*) $p<0,05$ & &
\end{tabular}

nas que componían inicialmente cada cohorte, el número de fallecimientos ocurridos durante el seguimiento y el total de personas/año a riesgo para cada subgrupo (discriminado por grupo de edad y presencia o no de algún factor de riesgo).

Globalmente observamos un total de 30 neumonías en CV y 18 neumonías en CNV, lo que implica una tasa de incidencia media anual de neumonía de $13,29 \%$ personas mayores de 65 años $(16,6 \%$ en $\mathrm{CV}$ y $9,96 \%$ en $\mathrm{CNV}$ ); el riesgo relativo para la adquisición de neumonía en CV fue, por tanto, de 1,67 (IC 95\%: 0,95-2,92).

El número observado de neumonías aumentó con la edad, siendo la incidencia media anual de neumonías entre la población de 65 a 75 años de $12,40 \%$ en $\mathrm{CV}$ y de $5,10 \%$ en $\mathrm{CNV}(\mathrm{p}=0,10)$, mientras que entre la población mayor de 75 años la incidencia media anual de neumonías fue de $23,58 \%$ en CV y 19,03\%o en CNV $(p=0,32)$. Del mismo modo el número observado de neumonías fue mayor cuando había presencia de factores de riesgo, obteniendo una tasa media anual de incidencia de neumonías de 4,22\% en personas sin factores de riesgo de la CV y 7,23\%o en personas sin factores de riesgo de CNV $(p=0,4)$, mientras que la incidencia entre personas con algún factor

Tabla III

\begin{tabular}{|c|c|c|c|c|c|c|}
\hline \multicolumn{7}{|c|}{ DESCRIPCIÓN DE LAS COHORTES POR GRUPOS DE EDAD FACTORES DE RIESGO } \\
\hline & \multicolumn{3}{|c|}{ Vacunados } & \multicolumn{3}{|c|}{ No vacunados } \\
\hline & $\begin{array}{l}\mathbf{N}^{0} \text { inicial de } \\
\text { personas }\end{array}$ & $\begin{array}{c}\text { Pérdidas por } \\
\text { defunción }\end{array}$ & $\begin{array}{l}N^{0} \text { personas/ } \\
\text { año a riesgo }\end{array}$ & $\begin{array}{c}\mathrm{N}^{0} \text { inicial } \\
\text { de personas }\end{array}$ & $\begin{array}{l}\text { Pérdidas por } \\
\text { defunción }\end{array}$ & $\begin{array}{l}N^{0} \text { personas/ } \\
\text { año a riesgo }\end{array}$ \\
\hline 65-75 años sin FR & 98 & 11 & 555 & 75 & 8 & 426 \\
\hline 65-75 años con FR & 90 & 9 & 513 & 121 & 13 & 687 \\
\hline Mayores de 75 sin FR & 60 & 23 & 291 & 40 & 14 & 198 \\
\hline Mayores de 75 con FR & 53 & 25 & 243 & 65 & 30 & 300 \\
\hline Total & 301 & 68 & 1.602 & 301 & 65 & 1.611 \\
\hline
\end{tabular}


de riesgo fue de $30,30 \%$ en CV y de $11,63 \%$ en CNV $(\mathrm{p}=0,07)$ (Tabla IV).

Si estratificamos por hábito tabáquico (única variable que presentaba diferente distribución en ambas cohortes) observamos 5 neumonías entre los 30 pacientes fumadores de la $\mathrm{CV}$ frente a 3 neumonías entre los 14 fumadores de CNV. Estos datos suponen una incidencia media anual de $27,78 \%$ en fumadores vacunados frente a un $35,71 \%$ en fumadores no vacunados, lo cual supone un RR de 0,78 en fumadores que hayan sido vacunados (IC 95\%:0,22-2,81).

Estos datos suponen un riesgo atribuible de $7,93 \%$ y , por tanto, el número de pacientes a vacunar mayores de 65 años necesario para prevenir una neumonía en fumadores (NNT) sería de 126.

En la tabla V puede verse la incidencia de neumonías en los diferentes grupos de estudio en función simultáneamente del grupo de edad y de la presencia de factores de riesgo. A destacar que la mayor incidencia de neumonías se produjo entre aquellas personas vacunadas que presentaban algún factor de riesgo (37,66\% en mayores de 75 años de CV y $25,91 \%$ en CV de 65 a 75 años)

En la tabla VI hemos calculado la incidencia de neumonías en cada uno de los años de seguimiento con objeto de valorar la disminución de la eficacia relacionada con el descenso de nivel de anticuerpos, sin observar tampoco diferencias significativas entre las tasas de incidencia durante el primer y segundo trienio de seguimiento.

Por último, no hemos observado ds al comparar el índice de letalidad de las neumonías en ambas cohortes, ya que éste fue del 13,7\% en CV frente a un $15,7 \%$ en CNV. Tampoco observamos ninguna ds al comparar de forma individual el resto de variables que componían la escala de severidad, siendo similar el número de pacientes de cada cohorte ( 7 en vacunados y 6 en no vacunados) en los que no se disponía de los datos necesarios para la valoración de la severidad.

\section{DISCUSIÓN}

A la vista de los resultados obtenidos resulta indudable que la incidencia de neumonías es mayor entre las personas que presentaban algún factor de riesgo (tanto en vacunados como no vacunados). Este hecho podría atribuirse no sólo a aspectos

Tabla IV

\begin{tabular}{|c|c|c|c|c|}
\hline \multicolumn{5}{|c|}{ INCIDENCIA DE NEUMONÍAS SEGÚN EDAD Y PRESENCIA DE FACTORES DE RIESGO } \\
\hline & \multicolumn{2}{|c|}{ Vacunados } & \multicolumn{2}{|c|}{ No vacunados } \\
\hline & $\begin{array}{c}\text { Tasa de incidencia } \\
\text { media anual } \\
1994-99\end{array}$ & $\begin{array}{c}\text { Densidad de incidencia } \\
\text { media anual } \\
1994-99\end{array}$ & $\begin{array}{c}\text { Tasa de incidencia } \\
\text { media anual } \\
1994-99\end{array}$ & $\begin{array}{c}\text { Densidad de incidencia } \\
\text { media anual } \\
1994-99\end{array}$ \\
\hline Total 65-74 años & 12,40 & 13,11 & 5,10 & 5,39 \\
\hline Total $\geq 75$ años & 23,58 & 29,96 & 19,03 & 24,11 \\
\hline Total sin FR & 4,22 & 4,73 & 7,23 & 8,01 \\
\hline Total con FR & 30,30 & 34,39 & 11,63 & 13,17 \\
\hline Total & 16,60 & 18,74 & 9,96 & 11,17 \\
\hline
\end{tabular}

Tasas expresadas por 1.000 habitantes.

Tabla V

\begin{tabular}{|c|c|c|c|c|}
\hline \multicolumn{5}{|c|}{ INCIDENCIA DE NEUMONÍAS DISCRIMINADAS POR GRUPOS DE EDAD Y FACTORES DE RIESGO } \\
\hline & \multicolumn{2}{|c|}{ Vacunados } & \multicolumn{2}{|c|}{ No vacunados } \\
\hline & $\begin{array}{c}\text { Tasa de incidencia } \\
\text { media anual } \\
1994-99\end{array}$ & $\begin{array}{c}\text { Densidad de incidencia } \\
\text { media anual } \\
1994-99\end{array}$ & $\begin{array}{c}\text { Tasa de incidencia } \\
\text { media anual } \\
1994-99\end{array}$ & $\begin{array}{c}\text { Densidad de incidencia } \\
\text { media anual } \\
1994-99\end{array}$ \\
\hline Sin FR (65-74) & 0 & 0 & 2,16 & 2,34 \\
\hline Con FR (65-74) & 25,91 & 27,29 & 6,83 & 7,27 \\
\hline Sin FR $(\geq 75)$ & 11,17 & 13,74 & 16,66 & 20,20 \\
\hline Con FR $(\geq 75)$ & 37,66 & 49,38 & 20,50 & 26,66 \\
\hline Total & 16,60 & 18,74 & 9,96 & 11,17 \\
\hline
\end{tabular}

Tasas expresadas por 1.000 habitantes. 
Tabla VI

\begin{tabular}{lcc}
\multicolumn{1}{c}{ TASAS DE INCIDENCIA DE NEUMONÍAS EN AMBAS COHORTES } \\
\hline & $\begin{array}{c}\text { Cohorte } \\
\text { vacunados }\end{array}$ & $\begin{array}{c}\text { Cohorte } \\
\text { no vacunados }\end{array}$ \\
& $13,29(4)$ & $3,32(1)$ \\
\hline Tim 1994 & $19,93(6)$ & $16,61(5)$ \\
Tim 1995 & $13,29(4)$ & $6,64(2)$ \\
Tim 1996 & $9,97(3)$ & $13,29(4)$ \\
Tim 1997 & $13,29(4)$ & $9,97(3)$ \\
Tim 1998 & $29,90(9)$ & $9,97(3)$ \\
Tim 1999 & $99,66(30)$ & $59,80(18)$ \\
Tia 1994-99 &
\end{tabular}

Tim: tasa de incidencia media anual: Tia: tasa de incidencia acumulada; tasas expresadas por 1.000 habitantes.

etiológicos ${ }^{20}$ de las neumonías observadas si no también a que la CV presentara unos mayores niveles de comorbilidad en cuanto a presencia simultánea de factores de riesgo, lo cual podría aumentar la susceptibilidad de adquisición de neumonías.

Debemos comentar el hecho de que nuestro estudio, a pesar de realizar un seguimiento longitudinal durante seis años a dos cohortes, éste se ha realizado de forma retrospectiva debido fundamentalmente a problemas logísticos, organizativos y de personal posteriores al diseño del inicial programa de vacunación implementado en 1993. Este hecho ha conllevado, entre otras cosas, a que carezcamos de la filiación de la etiología de la mayoría de las neumonías, así como del registro de algunos de los parámetros utilizados como indicadores de severidad en algunas neumonías (las que fueron diagnosticadas y tratadas exclusivamente desde APS) $(45,8 \%)$. En nuestra opinión, el primer déficit (ausencia de filiación etiológica) no resulta excesivamente relevante puesto que en la mayoría de estudios que valoran incidencia de neumonía adquirida en la comunidad (incluso estudios hospitalarios) no puede establecerse la etiología de la mayor parte de neumonías estudiadas. Por este motivo en nuestro estudio, tratándose de neumonías adquiridas en la comunidad, deberemos asumir que en correspondencia con datos bibliográficos de nuestro medio4-6, la frecuencia de neumonía neumocócica debe situarse alrededor del 20\% aunque la prevalencia de gérmenes diferentes al neumococo (Legionella, A. aureus, bacilos gram negativos) está aumentando 24 y es probable que el espectro etiológico de los casos severos se haya modificado durante los seis años de duración del estudio.

En relación a los parámetros utilizados como indicadores de severidad, debemos señalar que, si bien existen escalas validadas para valorar la gravedad potencial de una neumonía (y decidir así su ingreso hospitalario o no $)^{25}$, no encontramos sin embargo una escala satisfactoria que permitiera evaluar la severidad real de una neumonía instaurada, motivo por el cual decidimos utilizar el índice de letalidad y los parámetros de las cuatro dimensiones descritos en la metodología (constantes vitales, laboratorio, radiología y evolución clínico-terapéutica) como indicadores indirectos de severidad. El hecho de considerar como negativo o ausente cualquier parámetro del que no constaba registro en las historias clínicas (aunque puede introducir un cierto sesgo de información) puede resultar bastante acorde con la realidad suponer que si un parámetro no estaba registrado se debía al hecho de que el paciente no lo presentaba.

En relación a la asignación de los pacientes a ambas cohortes, si bien se trata de un estudio no experimental y consecuentemente sin asignación aleatoria, el hecho de no encontrar diferencias significativas en la composición de las dos cohortes conduce a pensar que no hubo excesivo sesgo de selección a pesar de que la asignación no fue aleatoria.

Sobre los resultados concretos obtenidos en nuestro estudio, cabe resaltar, en primer lugar que la incidencia anual de neumonías observadas fue globalmente de 13,29\%o en personas mayores de 65 años. Esta incidencia resulta sólo ligeramente inferior a la incidencia esperada de neumonías en este grupo de edad ${ }^{1-6}$, dato éste que apoya la rigurosidad y exhaustividad en cuanto al reclutamiento de las neumonías acaecidas durante el periodo de seguimiento, puesto que no existen datos que permitan suponer que la incidencia de neumonías en nuestra ABS pueda ser esencialmente diferente a la incidencia en nuestro medio.

No hemos podido evidenciar un efecto protector de la VAN en CV respecto a CNV ante la infección (coincidiendo con los metaanálisis más recientes) ${ }^{16,27}$. La incidencia de neumonías ha sido ligeramente superior en la cohorte de vacunados en relación a la de no vacunados, aunque deben tenerse en cuenta que si bien el riesgo relativo ha sido de 1,67 para la cohorte vacunada en relación a la no vacunada, pese a ser superior a la unidad no puede considerarse como significativo puesto que su IC al $95 \%$ oscila entre $0,95-2,92$.

Como es de esperar, la incidencia de neumonías aumenta claramente con la edad, aunque sorprendentemente sin existir diferencias significativas por estado vacunal.

La presencia de factores de riesgo resultó relevante puesto que el número observado de neumonías fue mayor cuando había presencia de alguno de ellos, aunque si bien la vacuna resulto eficaz para prevenir la infección entre aquellos pacientes que no presentaban ningún factor de riesgo $(\mathrm{p}=0,04)$ no pudimos evidenciar este mismo efecto protector, salvo en el caso del tabaco, para aquellos pacientes 
vacunados y que presentaban uno o más factores de riesgo.

Así, el análisis estratificado muestra que la vacuna podría prevenir claramente la infección entre los fumadores, puesto que pese a no resultar estadísticamente significativa observamos una importante reducción de la incidencia de neumonías entre los fumadores vacunados $(27,78 \%)$ frente a los fumadores no vacunados $(35,71 \%)$. Este hecho podría avalar la recomendación de vacunar a este subgrupo de pacientes.

Ha resultado llamativo observar que no se produjo este mismo efecto protector entre el subgrupo de pacientes diagnosticados de EPOC. Creemos que ello tal vez podría deberse a un posible sesgo de información, puesto que no dispusimos de una espirometría que permitiese clasificar el estadio de los EPOC. Es probable que los EPOC severos estuvieran incluidos en cohorte vacunada, mientras que aquellos catalogados como no severos estuvieran en la cohorte no vacunada.

Tampoco se ha evidenciado un efecto protector de la vacuna para disminuir la severidad de las neumonías presentadas en la cohorte vacunada. Es posible que este dato no adquiera significación estadística puesto que el estudio está realizado en un área básica con una población pequeña y con cohortes limitadas, por lo tanto asumimos la escasa potencia que puede tener nuestro estudio para detectar pequeñas diferencias. En este sentido, cabe señalar que, si bien los últimos datos bibliográficos permiten poner en duda la efectividad de la vacuna para prevenir la infección ${ }^{13}$, las últimas revisiones sugieren que la vacuna podría resultar eficaz para prevenir la bacteriemia y reducir por tanto la severidad de los cuadros clínicos ${ }^{16,27}$.

En definitiva, nuestros resultados no son discordantes con la literatura previa (muy heterogénea) que muestran incidencias ligeramente superiores, similares y también inferiores en personas vacunadas ${ }^{28}$.
Por último, debemos señalar que la aportación principal de nuestro trabajo radica en el hecho de tratarse de un estudio realizado a nivel comunitario, con un periodo de seguimiento amplio y sobre una población muy concreta (mayores de 65 años), emplazado además en nuestro medio. Independientemente de la indudable eficacia teórica (desarrollo de anticuerpos a nivel individual), nuestro trabajo aporta además la valoración de la efectividad real a nivel comunitario de una vacunación sobre la cual se está recomendando su aplicación de forma sistemática a pesar de no existir datos incontrovertibles sobre su efectividad en la práctica, especialmente sobre este grupo de población.

Finalmente debemos señalar que si bien somos conscientes de las limitaciones metodológicas (cohortes retrospectivas) y de la existencia de un no despreciable error beta (tamaño de las cohortes), pensamos que nuestro estudio permite plantear una duda razonable sobre la posible efectividad y/o eficiencia de la vacunación antineumocócica sistemática en mayores de 65 años en nuestro país. Creemos que debieran abrirse líneas de investigación con diseños prospectivos y multicéntricos que permitieran evaluar de forma más definitiva la efectividad y la eficiencia de la vacunación antineumocócica sistemática en la población de más de 65 años sin factores de riesgo adicionales (que representan por cierto la inmensa mayoría de la población diana de esta intervención).

\author{
CORRESPONDENCIA: \\ Ángel Vila Córcoles \\ C/ Mozart, 26. \\ 43007 Tarragona \\ e-mail: avila@saptarra.scs.es \\ Tel.: 977240666
}

\section{Bibliografía}

1. Subdirección General de Prestaciones y Evaluación de Tecnologías Sanitarias. Ministerio de Sanidad y Consumo. Informe sobre vacuna neumocóccica. Med Clin (Barc) 1994; 10: 383-6.

2. Gil de Gómez Barragán MJ, Acítores Augusto JM. Vacuna antineumocóccica: ¿a quién hay que vacunar? FMC 1996; 3: 185-90.

3. Pahissa Berga A. Vacunación antineumocócica. Tema pendiente de nuestra sanidad. Med Clin (Barc) 1991; 13: 499501.

4. Cruz D, Planes A. Vacuna antineumocóccica: \& a quién hay que vacunar? FMC 1996; 3: 181-5.
5. Departament de Sanitat i Seguretat Social. Bulletí Epidemiològic de Catalunya 1995; 16: 102-10.

6. Departament de Sanitat i Seguretat Social. Generalitat de Catalunya. Llibre Blanc: bases per a la integració de la prevenció a la pràctica assistencial. Barcelona: Doyma, 1993.

7. European Study on Community-Acquired Pneumonia (ESOCAP) Committee. Guidlines for management of adult community-adquired lower respiratory tract infections. Eur Respir J 1998; 11: 986-91.

8. Dorca J, Bello S, Blanquer J, De Celis P, Molinos L, Torres, et al. Diagnóstico y tratamiento de la neumonía adquirida en la comunidad. Arch Bronconeumol 1997; 33: 240-6. 
9. Niederman MS, Mandell LA, Anzueto A, Bass JE, Broghton WA, Campbell GD, et al. Guidelines for the management of adults with community-adquired pneumonia. Diagnosis, assessment of severety, antimicrobial therapy, and prevention. Am J Respir Crit Care Med 2001; 163: 1730-54.

10. Álvarez MJ, Mayer MA. El dilema sobre el coste efectividad de la vacuna antineumocócica sigue abierto. MEDIFAM 2001; 3: 115-21.

11. Salleras L. Vacunación antineumocócica en la tercera edad. Vacunas Invest Pract 2000; 1: 55-8.

12. Plans P, Garrido O, Salleras L. Coste-efectividad de la vacunación neumocócica en Cataluña. Rev Esp Salud Pública 1995; 69: 409-17.

13. Forrester HL, Jahnigen DW, Laforce FM. Inefficacy of pneumococcal vaccine in a high risk population. Am J Med 1987; 83: 425-30.

14. Butler JC, Breiman RF, Campell JF, Lipman HB, Broome CV, Facklam RR. Pneumococcal polysacharide vaccine efficacy. An evaluation of current recommendations. JAMA 1993; 270: 1826-131.

15. Shapiro ED, Berg AT, Austrian R, Schroeder D, Parcells V, Margolis A, et al. The protective efficacy of polyvalent pneumococcal polysacharyde vaccine. N Eng J Med 1991; 325: 1453-160.

16. R Andreu Moore, Philip J Wiffen, Benjamin A Lipsky. Are the pneumococcal polysaccharide vaccines effective? Metaanalysis of the prospective trials. BMC Family Practice 2000; 1: 1 .

17. Puig Barberà J, Belenguer Barea A, Goterris Pinto M, Brines Benlliure MJ. Efectividad de la vacuna frente al neumococo en el anciano. Revisión sistemática y metaanálisis. Aten Primaria 2002; 30: 269-83.

18. Aller AI, Garjón FJ, Buisán MJ, Palacín JC. ¿Está justificada la vacunación contra el neumococo en mayores de 65 años? Aten Primaria 2002; 7: 433-7.

19. Center for Disease Control and Prevention: Recommenda- tions of the Advisory Committee on Innunization Practices, the American Academy of Pediatrics, and the Americademy of Family Physicians: use of reminder an recall by vaccination providers to increase vaccinations rates. MMWR 1998; 47: 715-7.

20. Center for Disease Control and Prevention: Adult immunization programs in nontraditional settings: quality standards and guidance for program evaluation-a report of the National Vaccine Advisory Committee. MMWR 2000; 49 (No. RR-1): 1-14.

21. Briss PA, Rodewald LE, Hinman AR, Shefer AM, Strikas RA, Bernier RR, et al. Task Force on Community Preventive Services. Reviews of evidence regarding interventions to improve vaccination coverage in children, adolescent, and adults. Am J Prev Med 2000; 18 (1) (Supl. 1): 97-140.

22. Arjimón JM, Jiménez, J. Métodos de investigación clínica y epidemiológica. $2^{a}$ ed. Barcelona: Harcourt, 1999. p. 350-1.

23. Gudiol F. Infecciones neumocócicas. En: Farreras P, Rozman C. Medicina Interna. 13 a ed. Barcelona: Doyma, 1997. p. 2266-98.

24. Álvarez Gutierrez FJ, García Fernandez A, Elías Hernández T, Romero Contreras J, Romero Romero B, Castillo Gómez J. Neumonías en pacientes mayores de 60 años no hospitalizados. Incidencia de gérmenes atípicos y evolución clínicorradiológica. Med Clin (Barc) 2001; 117: 441-5.

25. Fine MJ, Auble TE, Yealy DM, Hanusa BH, Weissfeld LA, Singer DE. A prediction rule to identify low-risk patients with community-adquired pneumonia. N Engl J Med 1997; 336: 243-50.

26. Fedson DS. Pneumococcal vaccination for older adults: the first 20 years. Drugs Aging 1999; 15 (Supl. 1): 21-30.

27. Salleras L1, Urbiztondo Ll, Fernández N, Comín E, Sánchez F, Batalla J, et al. Vacunación antineumocócica en las personas mayores. Med Clin (Barc) 2001; 116: 18-23.

28. American Medical Association. Prevention of pneumococcal disease: use of the pneumococcal polysaccharide vaccine. Quality Care Alert, 1999. 